\title{
Análisis de la difusión de programas educativos en los medios de comunicación del cantón Vinces, período julio 2014 -julio 2015
}

\section{Analysis of the dissemination of educational programs in the media of the Vinces canton, period july 2014 -july 2015}

Erika Ninibeth Bustamante Arevalo

Universidad Politécnica Salesiana, Ecuador

Elier González Martínez

Universidad Politécnica Salesiana, Ecuador

Autor para correspondencia: egonzalez@ups.edu.ec, ebustamante@ups.edu.ec

Fecha de recepción: 20 de Julio de 2016 - Fecha de aceptación: 30 de Agosto de 2016

\begin{abstract}
Resumen
Los medios de comunicación han sido parte de las transformaciones del mundo globalizado que muestra la necesidad de fomentar la educación a través de sus programaciones. Analizar los elementos que caracterizan la difusión de contenidos formativos dentro del cantón Vinces se convierte en la problemática planteada durante el siguiente trabajo investigativo. Todos estos contenidos y la forma en que llegan a las audiencias han sido modificados en el tiempo, sin embargo, la poca preocupación que tienen los dueños de los medios locales han provocado la decadencia en la producción de estas programaciones. Pretendiendo responder a interrogantes, no solo planteadas en la exploración, sino en toda una población que busca respuestas ante los contenidos que se difunden, le conceden gran importancia en el desarrollo de esta investigación. Este acercamiento a la realidad del cantón Vinces puede devenir un referente en los análisis de contenidos educativos en sus programaciones locales.
\end{abstract}

Palabras claves: medios de comunicación; programas educativos; difusión de programas educativos; formación; sociedad

\begin{abstract}
Media has been a crucial part of the changes that affect a globalized world in need of promoting education through their programming. Analyzing the characteristic elements of educational contents broadcasted in the city of Vinces becomes the main goal in the following research work. This type of contents and the way they are presented to the audience have been modified through the years, however, the local media owner's very little concern on the disseminated topics has leaded to the decay in production. The aim of this work consists in trying to answer questions asked, not only during the survey but for local people, on the poor content transmitted. The approach to the reality of Vinces, on media matters, may bear a reference point for future local media contents studies and analysis.
\end{abstract}

Key words: media; educational programs; broadcast contents; society 


\section{Introducción}

El mundo cambia constantemente, y es tarea de los medios de comunicación ajustarse a las nuevas necesidades de una sociedad donde la inmediatez y la veracidad en la obtención de la información se convierten en prioridad así como una ventaja competitiva. Informar y entretener, no son los únicos objetivos de los medios de comunicación, puesto que la educación forma parte importante en la formación de las sociedades actuales. Estos propósitos o fines se deben reflejar en sus diversos contenidos de difusión como se manifiesta en el Artículo 8 de la Ley Orgánica de Comunicación del Ecuador: Los medios de comunicación, en forma general, difundirán contenidos de carácter informativo, educativo y cultural en forma prevalente. Estos contenidos deberán propender a la calidad y ser difusores de los valores y derechos fundamentales consignados en la Constitución y en los instrumentos internacionales de derechos humanos (p. 16).

Al ser los medios de comunicación un instrumento de transmisión de mensajes masivos, se convierten en la plataforma precisa para que las empresas aprovechen los espacios televisivos, radiales y escritos e inserten programaciones que muchas veces, benefician a la entidad y no a la sociedad. Según Biagi (2009) "después de los escándalos de los programas concursos, la principal crítica que recibieron las cadenas televisivas era que fueron motivadas por las clasificaciones de audiencia" (p. 177). Esto quiere decir que los medios de comunicación muestran interés en lo que el público desea, mas no en lo que necesita. En este caso se habla, entre más rating 1 brinde un programa es mucho mejor, porque los intereses económicos en su mayoría se priorizan dentro de las empresas comunicativas.

Para Matín-Barbero (2010) "la televisión entraña no solo un aumento en la inversión económica y la complejidad de la organización industrial, también un refinamiento cualitativo de los dispositivos ideológicos" (p.209). De esta forma se podría decir que la audiencia de las diversas programaciones son atraídas a ellas de múltiples formas, ya sea por el ocio, los gustos e incluso desde los aspectos psicológicos o necesidades. La obtención de esta información se hace a través de un estudio de campo. Se trata de adquirir datos precisos de los deseos del público, para entonces crear un programa que vaya acorde a su realidad, permitiendo a las empresas comunicativas tener públicos más diversos que estén pendientes de las transmisiones, el cual cree estar provisto de nuevos conocimientos, una nueva forma de hacer las cosas.

Sin embargo, a la educación en los medios de comunicación se le confiere gran relevancia, y debe valorarse aún más si se realiza a través de ellos, ya que niños, jóvenes y adultos están conectados a sus frecuencias. Para Aguaded Gómez (2010) "el proceso de enseñanza-aprendizaje que determina la educación en medios de comunicación requiere una selección de contenido acorde con un modelo de aprendizaje" (p. 67), por esta razón la radio, la televisión, el diario, deben formar a través de sus contenidos ciudadanos con criterios, con creatividad; pero, qué tan reales son estas afirmaciones.

\footnotetext{
${ }^{1}$ Los rating proporcionan información acerca de la audiencia que los patrocinadores alcanzan con sus anuncios. Biagi, S. (2009). Impacto de los medios de comunicación. México. Editorial Cengage Leirning.
} 
El 1lamado "analfabetismo audiovisual2" se ha convertido en la realidad de muchos países, especialmente de América Latina, donde le dan más importancia a la desnudez del ser humano que a la propia formación educativa de los niños, jóvenes e incluso los mismos adultos. Para poder definir este término se ha tomado como punto de partida elementos psicológicos y evolutivos del hombre, el mismo que pierde la cualidad de entender los códigos y diferenciar el mundo real (Ballesta, 2002). Esto ha hecho que la función de los medios en cuanto a la formación vaya decayendo. Por esta razón se convierte en algo palpable dentro de nuestra sociedad (Aguaded, 2015).

Con la tecnología y las nuevas formas de enseñanza que día a día aparecen a nuestro alrededor, los medios de comunicación tienen la ardua tarea de ayudar a fomentar la educación a través de sus transmisiones. Los programas planteados por los productores de las empresas deben fomentar valores y habilidades que ayuden a crecer al ser humano en todos los aspectos.

Al incorporar cualquier medio de comunicación a la enseñanza debe promover o ayudar a que se produzca, al menos teóricamente, un aprendizaje en los receptores, pero ello no significa que su incorporación haya seguido criterios didácticos o haya tenido repercusión didáctica en el diseño curricular para el cual fue diseñado (Sánchez, 2015). Al referirnos a lo manifestado por el autor, la enseñanza en los medios de comunicación debe provocar un aprendizaje para el receptor. Sin embargo, cabe preguntarse si realmente los criterios bajo los cuales se han realizado son educativos. Para Sánchez (2015) no es así, estas empresas programan para la formación de sus audiencias.

Aguaded Gómez (2010) expresa que una buena propuesta educativa dentro de los medios de comunicación se debe centrar en estos aspectos:

- Las fuentes, los orígenes y los factores determinantes de las construcciones de los medios

- Las técnicas y los códigos empleados por los medios de manera predominante para convencer de la verdad de sus representaciones: rapidez, actualidad, color, movimiento, sonido, impacto, imagen.

- La naturaleza de la realidad construida por los medios y los valores implícitos en sus representaciones: engaño, espectáculo, belleza, lujo, estereotipos. (p. 67-68)

Existen muchos tipos de programaciones que han permitido a las audiencias escoger uno de ellos para ver, escuchar o leer. En Ecuador, concurren medios de comunicación que día a día informan, entretienen y educan. A pesar de las múltiples transformaciones que han sufrido los medios ecuatorianos siguen manteniendo la finalidad de informar, sin embargo, la aparición de nuevos canales, revistas, periódicos y radios han ampliado la gama de opciones para el público y la variedad de programas difundidos.

La Constitución del Ecuador, aprobada en el 2008, definió que la educación y la formación serían la base para poder emprender mejores procesos integrales. En este contexto la población se convierte en el eje transversal de los cambios, lo que conlleva a fortalecer los vínculos educacionales donde la enseñanza no formal hace parte de estos contribuyentes

\footnotetext{
${ }^{2}$ El analfabetismo digital es el desconocimiento de las herramientas de multimedia. Aguaded, J. (2010). La educación para la comunicación. La enseñanza de los medios en el contexto iberoamericano
} 
educativos. Al retomar la Ley Orgánica de Comunicación aprobada en junio del 2013, se propone un nuevo giro en la intervención del proceso periodístico, convirtiéndose en la primera ley que rige esta actividad. En la normativa se establecieron 119 artículos donde se crearon múltiples parámetros para una mejor calidad de la producción dentro de los medios de comunicación.

Uno de los puntos más representativos en la Ley Orgánica de Comunicación es el Art. 97 que hace referencia al espacio de la producción audiovisual nacional, la cual representa el $60 \%$ de la transmisión diaria de los medios del país. En cuanto a los espacios de contenido educativo el programa EDUCA fue una de las proyecciones que dio inicio a los cambios exigidos en los medios de comunicación de Ecuador. Este formato sirvió como piloto para crear espacios educativos a través de sus programaciones televisivas, radiales e impresas.

Para Zecchetto (2011) la educomunicación “...asume, entonces, la cara de una 'seducción comunicativa', entendimiento con esta expresión al conjunto atractivo de las experiencias compartidas de interacción" (p.43). Entonces se podría decir que de acuerdo a lo manifestado por este autor, la educación a través de los medios de comunicación conlleva un sin número de artes, una vez juntos, pueden lograr el objetivo de enseñar a toda la comunidad a través de una interacción que permita el contacto y enseñanza directa con el público.

El presente proyecto está dirigido a los pobladores del cantón Vinces, provincia Los Ríos, los cuales por muchos años, han buscado cambios y mejoras en la producción y trasmisión de la información dentro del cantón. En este proceso investigativo con la difusión de los productos educativos se pretende conocer y mostrar los motivos que tienen los medios de comunicación para la difusión de programas educativos y qué opinan sus pobladores al respecto.

En Vinces existen 6 medios de comunicación (televisivos: Tv París y RTV, radiales: Radio París, Radio Eiffel; impresos: La Crónica y La Noticia), los cuales pocas o nulas veces hacen emisión de productos educativos dirigidos a los habitantes de la región. Cabe destacar que esta investigación centrará su análisis en los medios televisivos y radiales. Los motivos son diversos, pero llegar al punto donde se concentra la problemática se convierte en prioridad para saber cuáles son los errores que se están cometiendo. Sin embargo, referenciar algunas soluciones frente a estos inconvenientes pudieran encontrarse también en el texto, aunque no sean el interés principal en la investigación.

Los medios de comunicación muchas veces en sus parrillas de programación han mostrado productos ya elaborados que están completamente aislados de la educación, convirtiéndose en un problema a ser resuelto. Sin dudas, la transmisión de programas educativos se convierte en una grave dificultad de los medios de comunicación de Vinces, situación a explicar en esta investigación. Es por ello que se pretende analizar los elementos que caracterizan la difusión de los programas educativos en los medios de comunicación del cantón Vinces durante el periodo julio 2014-julio 2015.

La intención principal se centrará en reconocer la cantidad de programas educativos y el análisis de su difusión en los medios locales del cantón Vinces. Se realizará análisis de las diferentes percepciones que tienen los dueños de los medios y los pobladores de las comunidades 
que forman el universo de esta investigación. Para los medios de comunicación del cantón Vinces, se pretende que propongan una mayor difusión de programaciones educativas para la población. Los contenidos se estiman que sean producidos por ellos mismos y no importados ya que en una sociedad donde la cultura formativa va en declive estos espacios mediáticos deben ayudar a afianzar los valores que se imparten desde casa. Los medios de comunicación deben cumplir cada uno de sus objetivos, informar, entretener y educar, sin preferir uno más que otro.

La importancia de la investigación consiste que el análisis a los programas educativos y su difusión en los medios de comunicación del cantón Vinces, constituye uno de los primeros acercamientos a esta problemática en la región. Además, este proyecto servirá de base para que los dueños de dichas empresas mejoren las producciones audiovisuales, con la finalidad de educar a la comunidad vinceña. Desde un estudio científico, con técnicas y análisis de resultados se pretende mostrar las falencias en cuanto a la difusión existentes dentro de los medios de comunicación del cantón Vinces.

\section{Metodología}

La investigación se realizó dentro de los parámetros explicativos, permitiendo así, una dirección a los estudios y las descripciones de terminologías relacionadas a la investigación. Además, se buscó aportar, desde la opinión social de los pobladores vinceños, elementos que incentiven la difusión de programaciones educativas para ayudar a la formación de niños, de jóvenes, adultos y ancianos. Se procedió a utilizar una metodología cualitativa, que permitió comprender el objeto de la investigación a través de opiniones, criterios en cuanto a los temas directos a explorar, información necesaria sobre los medios de comunicación, la difusión de programas educativos y su impacto en la población vinceña.

Pese a que la investigación es predominantemente cualitativa se necesitó implementar técnicas de la metodología cuantitativa, que sirvieron para recolectar datos estadísticos sobre las edades de los encuestados, los programas que se difunden en los medios de comunicación, el acceso que tienen a ellos, para luego ser interpretados desde una perspectiva cualitativa. Para Hernández, Fernández, \& Baptista (2010.) "el enfoque cuantitativo usa la recolección de datos, con base en la medición numérica y el análisis estadístico, para establecer patrones de comportamiento y probar teorías" (p.5). Esto consintió que la información obtenida con este método sirviera para comprobar y confirmar el poco interés que tienen las personas en la difusión de contenidos educativos en los medios del cantón.

Según Corbetta (2003) citado en Hernández, Fernández, \& Baptista (2010.) “el enfoque cualitativo evalúa el desarrollo natural de los sucesos, es decir, no hay manipulación ni estimulación con respecto a la realidad" (p. 9); lo que significa que la presente investigación fue una valoración enfocada en los programas televisivos y radiales, para mostrar la poca difusión en contenido educativo dentro de los medios de comunicación de Vinces sin interferir de manera directa en los datos que brindaron los actores sociales.

Para lograr un trabajo investigativo con buenas bases metodológicas fue necesario emplear el método teórico histórico-lógico, permitiendo la comparación de hechos trascendentales de los medios de comunicación, la importancia de la educación a través de ellos, así como la difusión de programaciones formativas que incentiven a la audiencia a prepararse. 
Dentro de la recopilación y producción de hechos referidos los temas de los medios de comunicación, programas educativos y difusión de estos, fue preciso emplear el método bibliográfico que posibilitó estimar teorías sobre la evolución de los medios de comunicación, investigaciones antes realizadas, datos demográficos del cantón Vinces. Además de conocer el estado y valoración actual de los programas formativos.

Otro de los métodos que se emplearon en la presente investigación fue el análisis de contenido. Esto permitió realizar una argumentación más profunda dentro del trabajo. Para Piñuel Raigada (2002): Se suele llamar análisis de contenido al conjunto de procedimientos interpretativos de productos comunicativos (mensajes, textos o discursos) que proceden de procesos singulares de comunicación previamente registrados, y que, basados en técnicas de medida, a veces cuantitativas (estadísticas basadas en el recuento de unidades), a veces cualitativas (lógicas basadas en la combinación de categorías) tienen por objeto elaborar y procesar datos relevantes sobre las condiciones mismas en que se han producido aquellos textos, o sobre las condiciones que puedan darse para su empleo posterior (p. 2).

Para realizar este método se procedió a sintonizar las frecuencias televisivas y radiales examinando tanto la programación como el contenido en los medios de comunicación del cantón. La observación participante es una de las técnicas que se emplearon en la presente investigación, con la intención de obtener datos consecuentes dentro de los medios de comunicación.

La expresión observación participante es empleada aquí para designar la investigación que involucra la interacción social entre el investigador y los informantes en el milieu de los últimos, y durante el cual se recogen datos de modo sistemático y no intrusivo. (Taylor \& Bogdan 1987, p. 31).

Como se muestra, la técnica permitió llegar a un conocimiento respecto a la situación de los medios de comunicación local y su falta de interés por el tipo de transmisiones de índole educativo. Esto facultó llegar a la raíz de un problema existente desde el momento en que estas empresas fueron creadas dentro de la comunidad vinceña. La aplicación de este procedimiento, se realizó de la siguiente manera: se confeccionó una guía de observación participante, después se procedió a visitar por tres semanas consecutivas las instalaciones de los medios de comunicación para conocer la metodología empleada para la difusión de sus programas y se estuvo en constante contacto con diversos grupos etarios para evidenciar las reacciones que tenían frente a las transmisiones televisivas realizadas.

La segunda técnica a utilizar fue la entrevista, en su contexto general, es una conversación mutua entre un entrevistador y un entrevistado, así lo afirma Galindo (1998) "por entrevista definimos, habitualmente, una conversación verbal entre dos o más seres humanos (entrevistador y entrevistado), cuya finalidad es lo que en verdad le otorga tal carácter" (p. 281).

Para elaborar esta técnica se empleó una sub-muestra o muestra suplementaria de manera intencional. Se aplicó a los dueños de los medios de comunicación del cantón, permitiendo una mayor cantidad de información. Esta técnica se utilizó de manera precisa, con el objetivo de comprobar si existe difusión de programaciones educativas en sus empresas. Se estableció en una guía de construcción personal y en el guión para las entrevistas se construyeron preguntas abiertas para que el entrevistado respondiera de manera libre. En las entrevistas se visitaron a los 
cuatro dueños de los medios de comunicación local (televisión y radio para obtener sus criterios. Previo a esto y para evitar contratiempos se procedió a llamar a los número telefónicos de las empresas para agendar la cita. Además las entrevistas fueron grabadas en audio y analizadas en el siguiente punto del artículo académico.

El enfoque cuantitativo utiliza la encuesta. Para Bueno (2003) "Es uno de los métodos que permite obtener información sobre fenómenos y procesos, que no pueden ser adquirida a partir de la observación directa ni de los distintos documentos existentes" (p.70). La obtención de datos estadísticos, tuvo un valor dentro del estudio de la difusión de los programas educativos en Vinces como elemento complementario en la recolección de información.

Dentro de lo social las encuestas permiten obtener estados de opinión, que van a servir para saber lo que realmente ocurre dentro de un sector. Por lo tanto los resultados arrojados en la investigación permitieron conocer la cantidad de transmisiones educativas existentes en estas empresas comunicativas, el acceso que tiene la comunidad del cantón a estos medios de comunicación y saber la cantidad aproximada de las personas consumidoras de tipo de presentaciones. En esta técnica fue necesario emplear cuestionarios escritos, aplicados a la muestra seleccionada. Además, las interrogantes estuvieron relacionadas a temas específicos, como la difusión de programas educativos, el acceso que tienen a ellos, entre otros. La recolección de esta información permitió cuantificar la cantidad de programas de índole educativa en los medios de comunicación local.

El cálculo de la muestra se declaró con un 95\% de confianza y un margen de error del $5 \%$, para un tamaño poblacional de 55.443 habitantes, dentro de ella se encontró diversos grupos etarios que formaron parte de esta selección. Para ello se recurrió a la siguiente fórmula del libro Estadística para la investigación social (Camarero, Almazán, Arribas, Mañas, \& Vallejos, 2010):

$$
\mathrm{n}=\frac{\mathrm{z}^{2}\left(\mathrm{p}^{*} \mathrm{q}\right)}{\frac{\mathrm{e}^{2}+\mathrm{z}^{2}\left(\mathrm{p}^{*} \mathrm{q}\right)}{\mathrm{N}}}
$$

Donde:

$\mathrm{n}=$ Tamaño de la muestra (55443)

$\mathrm{z}=$ valor asociado al nivel de confianza $(95 \%)$

$\mathrm{p}=$ Proporción de la población con las características deseadas (éxito 50\%)

$\mathrm{q}=$ Proporción de la población sin la característica deseada (fracaso 50\%)

$\mathrm{e}=$ Nivel de error dispuesto a cometer $(5 \%)$

De esta manera la muestra fue de 382 habitantes y los encuestados se escogieron al azar, permitiendo que los datos tengan una mayor confiabilidad y abarque una amplia gama de opiniones y criterios que ayudaron a profundizar los problemas existentes dentro de los medios de comunicación.

\section{Resultados}

Esta sección expone el análisis de las técnicas empleadas para lograr responder a los objetivos de la investigación. En primer lugar se presentan los resultados de la observación participante, en segunda instancia la entrevista y por última la encuesta. El diagnóstico realizado fue el hilo conductor en este proceso, logrando una triangulación entre las técnicas y elementos comunes dentro de la muestra seleccionada. En primera instancia se constató que la mayoría de las viviendas de las personas analizadas cuentan con el servicio de cable para la televisión. Esta 
es la única forma que tienen los moradores para acceder a los medios de comunicación del cantón. Además esta prestación es brindada por los dueños locales.

Los medios televisivos del sector evidencian poca difusión de contenidos educativos, con excepción del canal Rtv (Canal 3). Su parrilla de programación incluye noticieros, programas concursos y productos educativos realizados por ellos, con temas que aluden a la drogadicción dentro de los centros educativos del cantón. Tv París (Canal 6), no cuenta con programas educativos propios, a pesar que tienen noticieros, programas concursos y difunden películas. Cabe recalcar, que las instalaciones de este medio de comunicación están ubicadas en el mismo lugar que la radio París. En este último, existe un programa infantil donde se transmiten consejos a los padres sobre el cuidado de sus hijos, y sale al aire solamente los días sábados. Esta emisión se caracteriza por la interacción con el público a través de llamadas y mensajes.

Específicamente en la radio Eiffel, la programación es básica, existe un noticiero que se transmite en tres horarios, todos los días de la semana, con programas de entretenimiento, donde el público interactúa solicitando canciones u opinando del tema principal del día, además de difusiones completamente musicales. Sin embargo, Tv París (Canal 6); Rtv (Canal 3): Radio París Y Radio Eiffel tienen algo en común, transmiten todos los días (de lunes a domingo) en horarios de 15:00 a 16:00 el programa Educa, que es enviado por las instancias gubernamentales como parte de las políticas de difusión de contenidos educativos que actualmente maneja el gobierno de la Revolución Ciudadana. La entrevista se aplicó a los dueños de los medios de comunicación local. El objetivo fue conocer los criterios que tienen sobre los programas educativos incluidos dentro de sus parrillas de programación y algunas consideraciones sobre la producción de estos.

En primera instancia se indagó sobre la historia de los medios de comunicación del cantón. En el caso de las empresas televisivas, su creación se remonta a 10 años, primero surgió Rtv (Canal 3), y después Tv París (Canal 6). Mientras que las emisoras radiales poseen mucho más tiempo, con aproximadamente ya 15 años de existencia. La finalidad de su creación siempre fue la misma: informar a la comunidad vinceña y sus alrededores sobre los eventos que sucedían dentro y fuera del cantón. Al preguntar sobre el proceso de difusión de programas educativos se conoció que RTV desde su creación, agregó un elemento extra que permitió llegar aún más al público; además de las noticias, deportes y entretenimiento apreciados también en los otros medios, se preocupó por la educación de la ciudadanía, creando así, espacios formativos sobre temas que involucraban a todos (drogas, cuidado ambiental, valores, etc.).

Los programas transmitidos en este medio de comunicación tienen una duración máxima de 6 meses, analizan las problemáticas presentes para fomentar la educación de los vinceños a través de la pantalla televisiva local. Actualmente este medio tiene al aire un programa propio con el nombre: "Jóvenes en acción por un Vinces mejor", el cual pretende educar tanto a padres como a hijos sobre las consecuencias de estas sustancias en sus vidas. En el caso de las emisoras, radio París transmite un programa familiar, las locutoras son madre e hija y comparten con el público reflexiones sobre los valores dentro del hogar. Además, interactúan con la audiencia por medio de las redes sociales y llamadas telefónicas. 
Por otra parte, de acuerdo a la manifestado en el Art. 97 de la Ley Orgánica de Comunicación, todos los medios de comunicación locales (radiales y televisivos), deben realizar la difusión del programa Educa. Esta transmisión, se muestra al público de lunes a viernes, porque las instancias gubernamentales la envían los fines de semana para que sean pautadas en las programaciones habituales.

En cuanto a la poca inversión de programas educativos en los medios de comunicación, se manifestó que los factores que inciden en las transmisiones de temas formativos en las empresas son dos: el económico y el cultural. La publicidad en los medios de comunicación televisiva es poco rentable, ya que al ser un poblado pequeño, las personas que tienen negocios prefieren que la mercadería ofrecida por ellos se propague a través de folletos, redes sociales, etc. Esto se convierte en un factor indiscutible de la poca rentabilidad económica en las empresas de comunicación.

Las emisoras radiales tampoco se escaparon de esta realidad, los dueños de estos medios, hicieron comparaciones con grandes ciudades, como Guayaquil, Quito, para mostrar la brecha abismal que los diferencia, es decir, en Vinces el valor de las pautas son muy bajas y por ende la falta de herramientas es muy visible. Además, para ellos no contar con el apoyo de las instancias municipales es otro problema que no les permite avanzar. Según los dueños de los medios de comunicación, la Superintendencia de Información y Comunicación (Supercom) constantemente está incitándolos para que adquieran productos educativos a través de ferias de comunicación. Sin embargo, los costos de adquisición son elevados, manifestando que solo un programa de esta índole para radio está aproximadamente en 4000 dólares, lo que se convierte en uno de los motivos de poca inversión en este tipo de transmisiones.

Otro de los factores visibles que se manifestó durante estas entrevistas fue la concordancia de los dueños en decir que: "la sociedad vinceña está muy poco identificada con los programas educativos". La juventud, especialmente, se encuentra en medio de muchas herramientas tecnológicas que les permiten acceder a plataformas de acuerdo a sus gustos (youtube, redes sociales, etc.) y menos a espacios que fomenten su formación. Por esta razón, ellos prefieren darle al público lo que pide, ya que en caso contrario, se corre el riesgo de no ser sintonizados por la audiencia y sus ingresos bajen aún más, lo cual puede provocar el cierre del medio de comunicación.

Posteriormente se procedió a realizar preguntas sobre la profesionalización de los trabajadores. Los resultados arrojaron que la mayor parte de los colaboradores no contaban con los estudios necesarios para laborar en estas empresas y mucho menos estar al frente de producciones educativas. Con el objetivo de erradicar esta falta de preparación y de acuerdo a las disposiciones de la nueva Ley Orgánica de Comunicación, actualmente los dueños de los medios de comunicación local envían a sus colaboradores a talleres de profesionalización en el Servicio Ecuatoriano de Capacitación Profesional (SECAP). Ahí, se especializan en diversas áreas como: productores, locutores, presentadores y escritores lo cual reportará un beneficio a la sociedad vinceña al contar en sus medios con especialistas en las temáticas.

Por otro lado, al manifestarse los jefes de los medios sobre una posible solución al problema de la difusión de programas educativos existieron dos ideas diferentes. La primera 
coincide en el trabajo en equipo que se debería hacer, a través de la división de costos entre todos, para comprar diferentes paquetes educativos. La segunda variante, propone un trabajo en conjunto pero encaminado a la elaboración del programa con los recursos que ellos tienen. Lo novedoso de esta última propuesta es que tendría sus bases en ideas y sugerencias de los pobladores del cantón, permitiendo un producto que responde a las necesidades y particularidades de la región; sin embargo, todo permanece en ideas y no se visualizan proyectos conjuntos que respondan a una de las dos variantes.

La encuesta fue aplicada al total de la muestra, 382 moradores de los diferentes sectores del cantón Vinces. El objetivo de la encuesta fue conocer qué tanto sabe la población vinceña de los programas educativos difundidos en los medios de comunicación local, así como la cantidad transmisiones educativas sintonizadas en estos medios locales. Otro de los puntos fue determinar si la población identifica estas producciones. La aplicación de esta técnica mostró los siguientes resultados: la primera pregunta se planteó con la finalidad de conocer la edad de los moradores encuestados. Los resultados fueron muy variados, esto nos permitió obtener una mayor cantidad de criterios que posibilitaron alcanzar mejores resultados en el presente artículo. Las edades de los encuestados varían entre los 18 hasta los 73 años, siendo el sector joven el más representativo con un 43 por ciento del total encuestados, seguido por el adulto con 36 por ciento y el resto, personas con más de 60años.

Con la segunda interrogante se pretendió indagar sobre el acceso que tiene la población del cantón a los medios de comunicación local, ya sean televisivos o radiales. Como se muestra en la Tabla 1 el acceso a los medios de comunicación local, no es el problema dentro del sector. Estos números hacen visible la presencia inminente de los medios comunicativos en las vidas de los habitantes. En esta interrogante los datos reflejaron que el $84 \%$ si tiene acceso a los medios de comunicación, ya sean televisivos o radiales, mientras que el 16\% no lo tiene.

\begin{tabular}{ccc} 
Tabla 1. Personas con acceso a medios de comunicación local \\
\cline { 2 - 3 } Opción & Cantidad & Porcentaje \\
\hline SI & 321 & $84 \%$ \\
NO & 61 & $16 \%$ \\
Total & 382 & $100 \%$
\end{tabular}

Fuente: Elaboración propia

En consecuencia con el cuestionamiento anterior, se propuso conocer ¿cuánto gustan los pobladores del cantón de las programaciones que se transmiten en los medios de comunicación local? Las respuestas en esta pregunta mostraron una gran diferencia entre las personas que gustan o no de estas programaciones. La mayoría de los encuestados ( 90 por ciento) prefieren la televisión de otras cadenas de medios comunicativos, porque en los locales no existe variedad. La presencia de empresas como tv cable, Claro tv y Direct TV, ha hecho que los medios locales no sean sintonizados, porque en ellos la oferta de programaciones es menor. Por otro lado, canales nacionales como Ecuavisa, Tc Televisión, Teleamazonas, RTS, también se convierten en la competencia directa de las empresas locales. Los que gustan de estas transmisiones están motivados por saber lo ocurrido dentro del cantón. Sin embargo, una vez visto lo que buscaban, sintonizan otros canales, ya sea para ver novelas o películas. 
En cuanto a la radio explicaron que las programaciones si son variadas, pero poco son sintonizadas. Los jóvenes que participaron de esta encuesta, expresaron sus preferencias por emisoras nacionales, más que locales, ya que en ellas se emite música de acuerdo a sus gustos; en cambio en las del cantón la programación no se ajusta, según ellos, con las tendencias actuales de los más jóvenes. Por otro lado, las personas adultas fueron las que expresaron su agrado por las programaciones que se emiten en las radios del cantón, manifestando que las emisoras conservan las raíces de un pueblo que ha crecido.

El siguiente aspecto a indagar estuvo encaminado en saber si los habitantes dentro del cantón Vinces, conocen de la existencia o difusión de programas educativos. Los resultados arrojaron que el 88 por ciento de las personas encuestadas no han oído o visto programaciones educativas en los medios de comunicación local, pese a la accesibilidad que tienen a estos. La respuesta consensuada fue que en Vinces, los medios de comunicación no invierten en este tipo de productos que serían útiles para toda la sociedad, porque priman otro tipo de intereses.

La última interrogante estuvo enfocada en cuántos programas educativos han visto o escuchado por las personas encuestadas. Los resultados mostraron que, de haber reconocido en la pregunta anterior la existencia de estos programas, sólo han sido visto o escuchado una o dos transmisiones dentro de los medios de comunicación local con un 87 por ciento del total. Los datos arrojados son realmente preocupantes, ya que se convirtió en un referente de la poca transmisión de productos educativos en los medios de comunicación del cantón (Ver tabla 2). Para los encuestados la mayor cantidad de programas de esta índole en las empresas comunicativas locales, pertenecen al gobierno.

\begin{tabular}{ccc} 
Tabla 2. Cantidad de & programas educativos vistos \\
\hline Opción & Cantidad & Porcentaje \\
\hline $0-2$ & 371 & $87 \%$ \\
$3-5$ & 11 & $8 \%$ \\
$6-8$ & 0 & $5 \%$ \\
Total & 382 & $100 \%$ \\
\hline
\end{tabular}

Fuente: Elaboración propia.

\section{Conclusiones}

De acuerdo a los resultados de las técnicas empleadas en la presente investigación, se pudo constatar que los programas educativos no tienen una gran de relevancia en los medios de comunicación del cantón Vinces, dentro de las programaciones habituales no existe más de uno o dos programas con estas temáticas. La televisión como medio de comunicación local tiene 10 años de funcionamiento, mientras que la radio alcanza los 15 años de creación. Al ser empresas relativamente nuevas, el trabajo a realizar es arduo, convirtiéndose en una de las desventajas más predominantes frente a empresas de nivel nacional e internacional por la gama de programaciones que ellos ofertan al público y sus posiciones privilegiadas frente a las diferentes audiencias. Sin embargo, pese a las nuevas disposiciones comunicacionales, se han ido reinventando de a poco, pero esto no ha sido suficiente, porque aún existen necesidades y carencias dentro de ellas.

Desde su creación, para acceder a la televisión en el cantón, se tiene que contratar un servicio de cable, el cual es brindado por los dueños de estos medios locales. En el caso de la 
radio deben ser vendidos grandes laxos de tiempo para publicidad porque es una fuente de ingreso importante para el sector. Esto les permite la obtención de ingresos que les otorguen mantenerse al aire. A decir de los dueños, el poco interés de los pobladores en temas educativos podría disminuir los índices de audiencia y por consecuencia los beneficios que se derivan de esta actividad, siendo este aspecto el principal temor de ellos.

Por otro lado, la existencia de pocos programas educativos dentro de los medios de comunicación del cantón fue una respuesta consensuada entre los dueños de las empresas y los pobladores encuestados. A pesar de que la Ley Orgánica de Comunicación estimula la creación de espacios para este tipo de programas, no existe una concientización en los pobladores y los propietarios de los medios de comunicación de la importancia social que reviste difundir estos contenidos. El programa Educa es la principal muestra de lo antes expuesto, las empresas transmiten en sus programaciones los diferentes tópicos que vienen incluidos en el paquete audiovisual. Cabe recalcar que, tanto en la televisión como la radio, estas transmisiones se difunden por disposiciones gubernamentales, más no por iniciativa propia del medio comunicativo.

Existen alrededor de ocho presentaciones de las cuales una es realizada por los trabajadores de las empresas comunicativas del cantón. Rtv (Canal 3), es el único medio televisivo que realiza programas educativos. Las transmisiones propias de los medios de comunicación, en este caso RTV, tiene una planificación antes de mostrarse al público. Ellos analizan la situación más relevante que se vive en el cantón y de acuerdo a eso realizan un programa educativo, el cual cuenta con la participación de doctores y autoridades; sin embargo, estas transmisiones, de acuerdo a la observación que se realizó, tienen muchas falencias. Los presentadores especialmente no tienen fluidez de ideas y constantemente preguntan lo mismo. La interacción con el público es muy importante, no obstante solo se promueve a través de las redes sociales, mensajes de textos u otras herramientas tecnológicas.

Diferentes causas fueron encontradas durante el proceso investigativo para la escasa difusión de contenidos educativos; la poca experiencia en cuanto a la producción de programas de esta índole constituye una de ellas, y es considerada como uno de los principales motivos para que no realicen productos comunicativos con esta temática. La experticia y profesionalización de los trabajadores es un tema a valorar. Los resultados arrojaron deficiencias en este aspecto, componente esencial para lograr las metas planteadas en cualquier programa, no solo educativo. Sin embargo, frente a esta problemática se están tomando soluciones que ayudarán a mejorar las transmisiones en los espacios tanto de televisión como de radio. Los dueños de las empresas manifestaron durante las entrevistas que para erradicar la presencia de personas, sin conocimientos de producción, se envían a los trabajadores a cursos de superación y preparación en las diversas áreas laborales.

De acuerdo a lo manifestado por personas entrevistadas, los programas son simples copias de productos ya elaborados y no mantienen la curiosidad y la atención del televidente o del radioescucha. La poca o nula aceptación que tienen los programas de producción local ha devenido en un obstáculo para consolidación y desarrollo de estos medios según parámetros establecidos y de competencia. Durante el proceso investigativo se pudo constatar que tanto la televisión como la radio local, no cuentan con medición de rating en los horarios donde se 
trasmiten los programas educativos. Por consiguiente se puede concluir a priori, que los dueños de los medios no tienen una fuente confiable para saber la aceptación de estos programas, o sea, están emitiendo criterios sobre la base de ideas prestablecidas. Al entrelazar la información anterior con lo manifestado por los encuestados, se aprecia que existe una falta de preocupación por parte de los encargados en producir programas educativos que respondan a los estándares más elevados de calidad.

Los programas educativos en lugar de ser un espacio de ayuda para la sociedad, se convierten en un tema de pura formalidad ante lo establecido por la ley, más no por la importancia de sus mensajes a la comunidad. Más allá de los intereses económicos debe primar una filosofía que promueva una televisión más sana y que genere en los pobladores verdaderos sentimientos de responsabilidad ciudadana. Los medios de comunicación del cantón Vinces, como cualquier otra empresa enfrentan situaciones que día a día se tienen que superar. La falta de producciones educativas dentro de los medios de comunicación local es algo que debe ser tratado con mayor profundidad. Estas empresas deben fomentar la educación de sus audiencias, convirtiéndose así, en un referente formativo para la sociedad.

\section{Bibliografía}

Aguaded Gómez, J. I. (2010). La educación para la educación. Una alfabetización para un mundo global. En M. E. Moral, Televisión: desarrollo de la creatividad e infancia (págs. 55-77). Barcelona: Octeadro.

Aguaded, J. (27 de agosto de 2015). Grupo Comunicar. Obtenido de http://www.grupocomunicar.com/contenidos/pdf/educacion-y-medios-de-comunicacion/05aguaded.pdf

Ballesta Pagán, J. (2002). Medios de comunicación para una sociedad global. Murcia: Universidad de Murcia.

Bernal, C. (2010). Metodología de la investigación. Colombia: Pearson Educación.

Biagi, S. (2009). Impacto de los medios de comunicación. . México: CengageLeirning.

Bueno, E. (2003). La investigación científica. Teoría y metodología. . Zacateca-México: Universidad Autónoma de Zacateca.

Galindo, J. (1998). Técnicas de investigación, en sociedad, cultura y comunicación. México: Pearson.

Hernández, R., Fernández, C., \& Baptista, P. (2010.). Metodología de la Investigación. México, D. F.: McGraw Hill.

Matín-Barbero, J. (2010). De los medios a las mediciones. . Barcelona: Antropos. .

Piñuel Raigada, J. L. (2002). Recuperado el 10 de Diciembre de 2015, de file:///C:/Users/Lab\%20Multimedios/Downloads/268-2013-07-29-

Pinuel_Raigada_AnalisisContenido_2002_EstudiosSociolinguisticaUVigo\%20(1).pdf

Rodríguez, G., Gil, J., \& García, E. (1998). Metodología de la investigación cualitativa. La Habana: Félix Varela.

Sánchez, F. M. (11 de Diciembre de 2015). Edutec . Obtenido de http://edutec.rediris.es/documentos/1999/televis.htm

Taylor, S., \& Bogdan, R. (1987). Introducción a los métodos cualitativos de investigación. Barcelona: Paidós Básica.

Zecchetto, V. (2011). Educomunicación. Quito-Ecuador: Abya-Yala. 